\title{
A closer look at the influence of tubular initial conditions on two-particle correlations
}

\author{
R.P.G.Andrade, F.Grassi, Y.Hama, and W.-L.Qian \\ Instituto de Física, Universidade de São Paulo, SP, Brazil
}

(Dated: Oct. 2009)

\begin{abstract}
In a recent paper, the hydrodynamic code NEXSPheRIO was used in conjunction with STAR analysis methods to study two-particle correlations as function of $\Delta \eta$ and $\Delta \phi$. The various structures observed in data were reproduced. In this work, we discuss the origin of these structures as well as present new results.
\end{abstract}

\section{INTRODUCTION}

One of the most striking results in relativistic heavy ion collisions is the existence of some structures in the two-particle correlations [1, 2, 3]. One structure has a long (pseudo)rapidity extent [4] and a narrow azimuthal extent. The other may have a long (pseudo)rapidity extent and has a single or double hump in azimuth. In order that two-particles A and B emitted at some proper time $\tau_{\text {f.out }}$ appear as correlated, the process that correlated them must have occurred[5, 6] at some proper time $\tau \leq \tau_{\text {f.out }} \exp \left(-\left|y_{A}-y_{B}\right| / 2\right)$. Therefore, the existence of long range (pseudo)rapidity correlations must be related to early times in the nuclear collisions.

These two-particle azimuthal correlations data have motivated many theoretical investigations (for a short critical review see e.g. [7]). In many of these approaches, the mechanism is closely related to jet quenching and the response of the medium to the deposited energy. However, 1) the ridge structure is also seen at low transverse momentum [8, 9]; 2) recent experimental data seem to indicate no correlation between high pt trigger and associated ridge particles [10]. In another class of models, it is suggested that the combined effect of a longitudinal structure in the initial conditions (IC) and transverse expansion is responsible for the ridge [5, 6, 11]. In this line, we have studied the two-particle correlation by using a hydrodynamic code NEXSPheRIO[12]. Both the near-side and double-hump away-side structures were reproduced. In this work, we discuss how exactly these structures appear as well as present new results.

\section{RIDGES AND PEAK IN NEXSPHERIO}

The NeXSPheRIO code uses IC from the microscopic code NeXus [13] and solves the hydrodynamic equations with the SPheRIO code [14], on an event by event basis. Figure 1 shows a typical example of IC with tubular structures along the collision axis. In our model, this is what causes the long (pseudo)rapidity extent of the two-particle correlations. We note that in figure 1, the near-side structure is composed of a ridge and a peak, as seen experimentally. In our approach, the IC constructed by "thermalizing" NeXus output [14] do not explicitly involve jets, these are however not totally forgotten in the IC as they leave some localized region with higher transverse fluid velocity as shown in figure 2. This region is not correlated with a tube so peak and ridge are independent. 

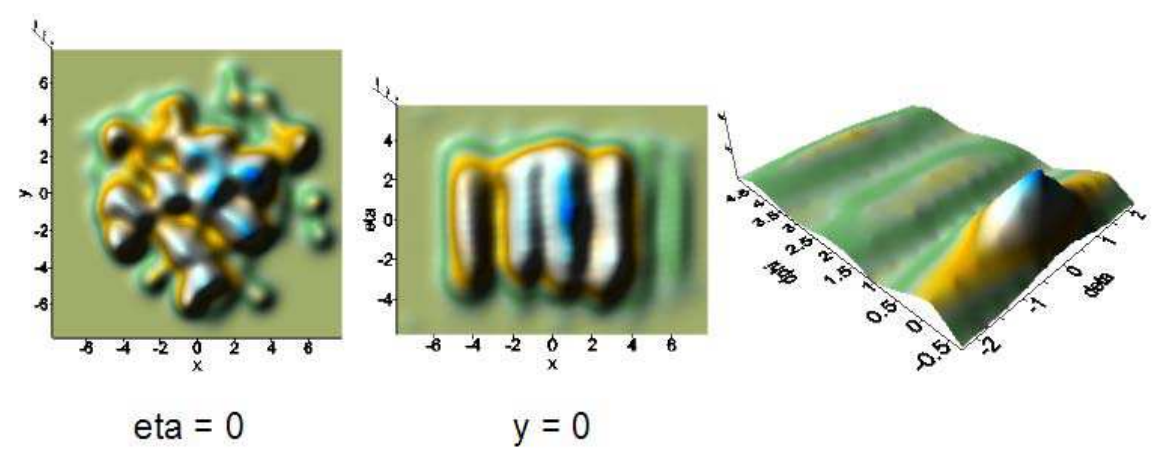

FIG. 1: NeXSPheRIO initial energy density in the transverse (left) and reaction (center) planes for a central $\mathrm{Au}+\mathrm{Au}$ collision at $200 \mathrm{GeV} \mathrm{A}$. Two-particle correlation (right).

We also note in figure 1 the existence not only of the near-side ridge but also of the doublehump away-side ridge. This result was obtained in [12] using the same methods as in the STAR analysis, in particular elliptic flow was removed using ZYAM. In this work, a totally different analysis is done, in particular the directions of the different event planes (which is calculable in our model[15]) are aligned, then the mixed event contribution is obtained and subtracted from the raw correlation. By doing this, the effects of $\eta$ distribution shape and flow are removed in a single step.

The fact that this new analysis leads to similar results as in [12] reinforces both approaches. However it is still unclear what causes in detail the near and away-side structures as well as what fixes the position of the double-hump in the away-side ridge.
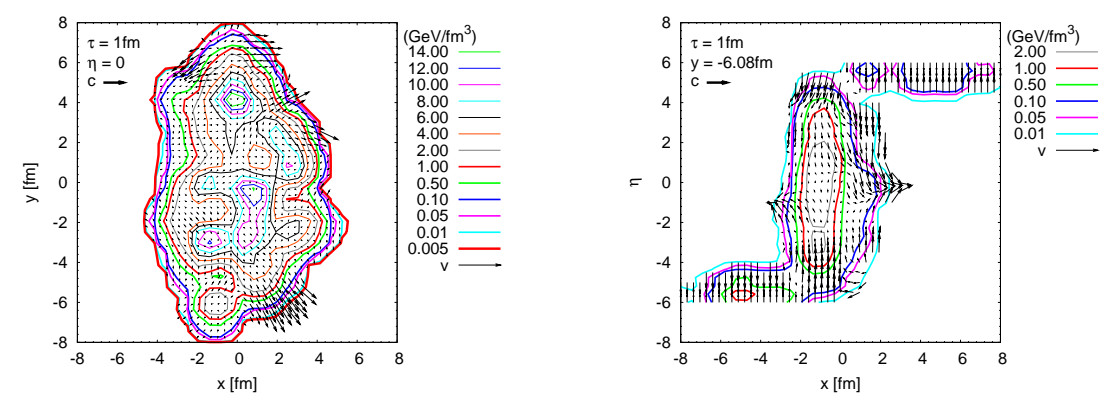

FIG. 2: NeXSPheRIO initial fluid velocity for a $\mathrm{Au}+\mathrm{Au}$ collision at $200 \mathrm{GeV}$ A in the $25-35 \%$ centrality window.

\section{ORIGIN OF THE RIDGES IN A SIMPLIFIED MODEL}

To investigate the origin of the ridges, we use a simplified two-dimensional model. This model consists of a slice of matter which initially has a high energy density spot in a smooth background. This slice subsequently undergoes transverse expansion and boost-invariant longitudinal expansion. More details are given in [16]. The single particle angular distribution has not a single peak as one might expect but two peaks located on both sides of the position of the tube as seen in figure 3 (left). This double peak structure is observed for all transverse momenta at more or less the same position [16] and its location is in agreement 

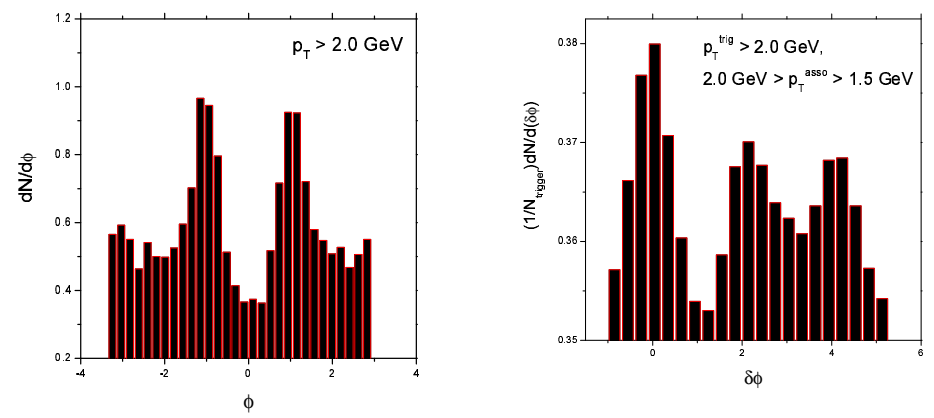

FIG. 3: Single (left) and two (right) particle angular distribution in the simplified model.

with data (for a central collision). The reason, as seen in figure 4 is that the effect of the tube is to deflect the otherwise isotropic radial flow.

From figure 3 (left), we can guess how the two-particle angular correlation will be. The trigger particle is more likely to be in one of the two peaks. We first choose the left-hand side peak. The associated particle is more likely to be also in this peak i.e. with $\Delta \phi=0$ or in the right-hand side peak with $\Delta \phi \sim+2$. If we choose the trigger particle in the right-hand side peak, the associated particle is more likely to be also in this peak i.e. with $\Delta \phi=0$ or in the left-hand side peak with $\Delta \phi \sim-2$. So the final two particle angular correlation must have a large central peak at $\Delta \phi=0$ and two smaller peaks respectively at $\Delta \phi \sim \pm 2$. Figure 3 (right) shows that this is indeed the case. The peak at $\Delta \phi=0$ corresponds to the nearside ridge and the peaks at $\Delta \phi \sim \pm 2$ form the double-hump ridge. We have checked that this structure is robust by studying the effect of the height and shape of the background, initial velocity, height, radius and location of the tube [16]. We are extending this model to non-central collisions.
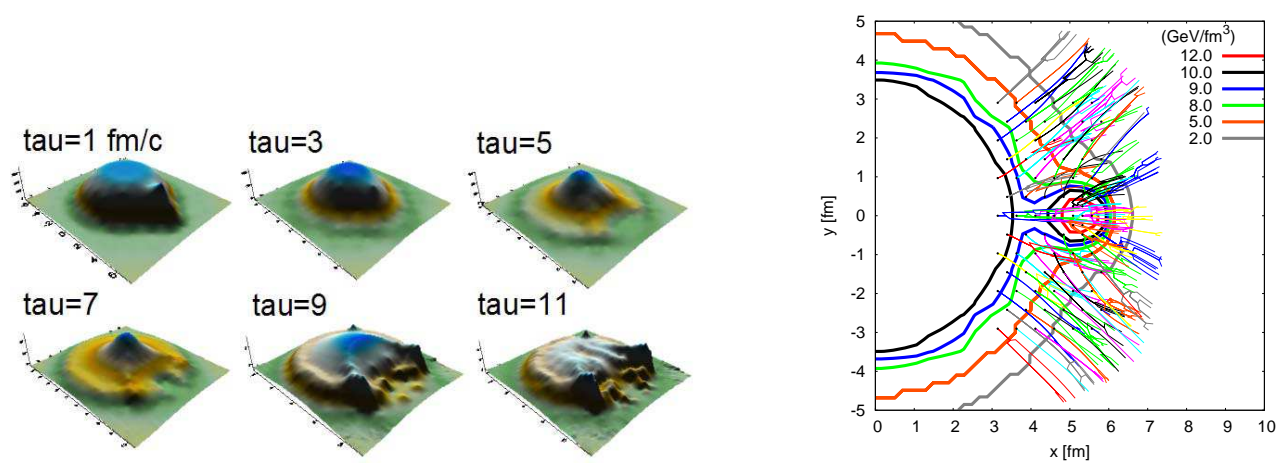

FIG. 4: Temporal evolution of energy density for the simplified model (left). Trajectories of the fluid cells around the tube (right).

\section{COMPARISON OF NEXSPHERIO RESULTS WITH EXPERIMENTAL DATA}

Now that the origin of the ridges is clarified, we return to a comparison of NeXSPheRIO results with data. Since, as already explained, the jets are "thermalized" in our model, a 


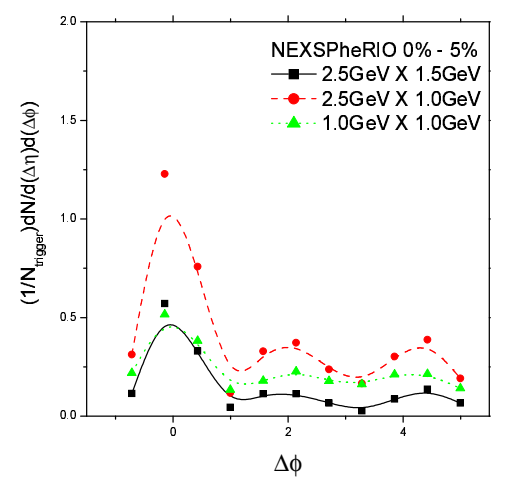

FIG. 5: NeXSPheRIO dependence of the ridges on the $p_{t}$ cutoffs for $\mathrm{Au}+\mathrm{Au}$ collisions at 200A $\mathrm{GeV}$.

precise quantitative comparison at this stage is not possible. Nevertheless several qualitative comparisons can be done.

For fixed $p_{t}^{\text {trig }}$ and increasing $p_{t}^{a s s o c}$, the near-side and away-side peaks decrease as seen in figure 5 for central collisions (this is generally expected since the number of associated particles decreases). On the other side, for fixed $p_{t}^{a s s o c}$ and increasing $p_{t}^{\text {trig }}$, the peaks increase. This behavior is in agreement with data (fig. 36 in [17]).

When going from central to peripherical collisions, the near-side ridge decreases and the away-side ridge changes from double to single hump, as seen in figure 6 and in conformity with data(fig. 36-38 in [17]).

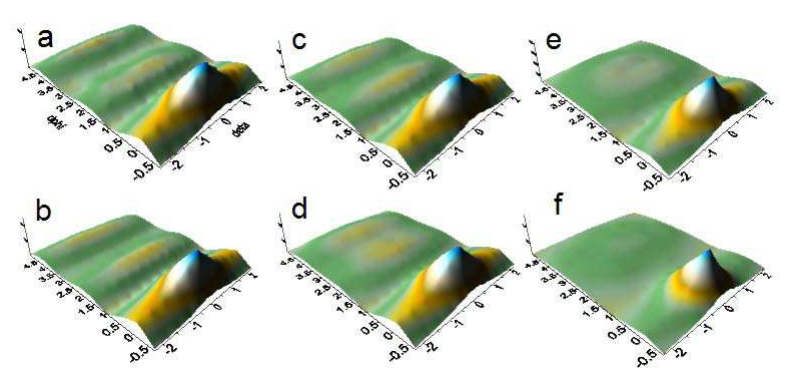

FIG. 6: Two-particle correlation, as computed with our NeXSPheRIO code, for different centrality windows of $\mathrm{Au}+\mathrm{Au}$ collisions at $200 \mathrm{~A} \mathrm{GeV}$ (a: 0-5\%, b: 5-10\%, c: 10-20\%, d: 20-30\%, e: 30-40\%, f: $40-50 \%) \cdot p_{t}^{\text {trig }}>2.5 \mathrm{GeV}$ and $p_{t}^{\text {assoc }}>1.5 \mathrm{GeV}$.

The correlation can be studied as a function of the trigger particle angle with relation to the event plane. In figure 7 for a mid-central window, the away-side ridge changes from single peak for in-plane trigger to double peak for out-of-plane trigger. For central collisions (not shown), it is always double-peaked. This is in accordance with data (fig.1 in [18]).

Some other qualitative features can also be mentioned. The near-side ridge seems to be present for untriggered correlation as found experimentally [8, 9] but our analysis method is different from the experimental one and this result is still being checked. As explained above, the near-side ridge is independent of the jet peak, also in agreement with data [10]. Due to its origin, we expect the near-side ridge to have similar composition as the bulk and 


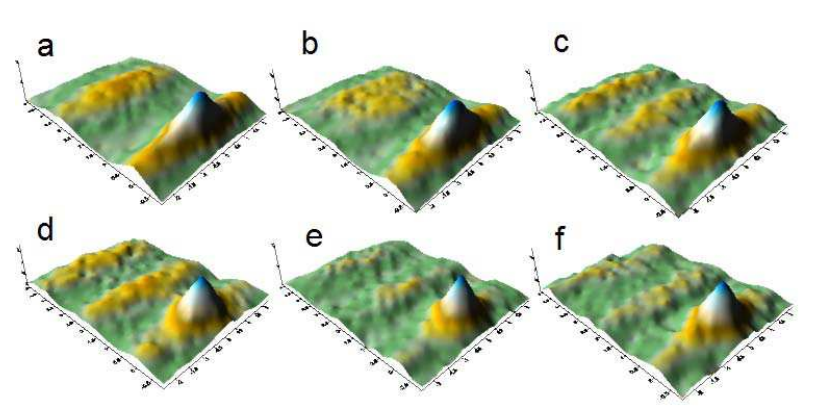

FIG. 7: Two-particle correlation, from NeXSPheRIO, for 20-30\% centrality $\mathrm{Au}+\mathrm{Au}$ collisions at $200 \mathrm{~A} \mathrm{GeV}$, for different $\phi_{s}\left(\mathrm{a}: \phi_{s}=0^{\circ}-15^{\circ}, \mathrm{b}: \phi_{s}=15^{\circ}-30^{\circ}, \mathrm{c}: \phi_{s}=30^{\circ}-45^{\circ}\right.$, d: $\phi_{s}=45^{\circ}-60^{\circ}$, e: $\phi_{s}=60^{\circ}-75^{\circ}$, f: $\phi_{s}=75^{\circ}-90^{\circ} . p_{t}^{\text {trig }}>3$. GeV and $p_{t}^{\text {assoc }}>1 . \mathrm{GeV}$.

its spectra should be a little harder than bulk (see figure 3 left and figgure 5 of [16]), also in line with data [10, 19]. We expect a similar behavior for the away-side ridge in accordance with data when available [10, 20]. Finally, for non-central collisions, due to their origin, we expect asymmetry in the ridges azimuthal correlation as function of the trigger angle with respect to the event plane; for the near-side ridge this has been observed in [21].

\section{CONCLUSION}

In conclusion, the hydrodynamic expansion starting from fluctuating tubular IC produces the various structures observed in the two-particle correlations [12]. We showed in this paper explicitly how this happens, using a simplified single tube model: this tube deflects matter in two directions which results in the near-side and away-side ridges. In addition we show that NeXSPheRIO code reproduces several other observed characteristics of the two-particle correlations.

Other models have been suggested to explain some of theses data. Three particle correlations might provide a way to discriminate: some models such as the Mach cone one predict that the associated particles emerge at two angles leading to figure 8 (left) while others such as the deflected jet model result in particles (if not flying with the trigger) emerging at one angle giving rise to figure 8 (right). Such a figure is also expected for the simplified one tube model and approximately for NeXSPheRIO central collisions. We are working on a precise prediction and comparison with data [22] .
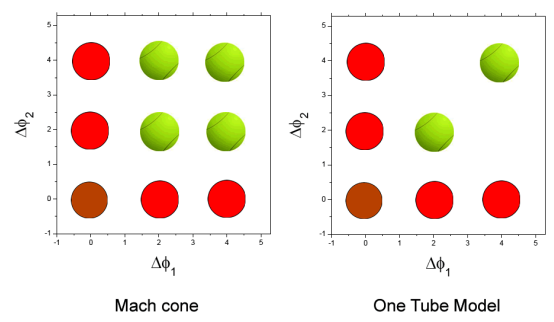

FIG. 8: Schematic three particle correlations as function of the angles of the two associated particles with respect to the trigger particle. 
It seems that an interesting observable would be $2+1$ correlations (first associated particle fixed in a narrow kinematical interval), which requires less statistics and leads to a clean prediction. For example choosing the trigger from one peak in figure 3 (left) and the first associated particle from the other peak, the second associated particle will be from any of the two peaks. This means that in two particle correlation between the second associated particle and the trigger presented in terms of $\Delta \eta$ and $\Delta \phi$, there would be two stripes. In contrast, the Mach-cone model would lead to three stripes.

\section{ACKNOWLEDGMENTS}

We thank for discussions with Takeshi Kodama, Jun Takahashi, Bernardo Tavares, Fuqiang Wang, Guoliang Ma, Paul Sorensen, Klaus Werner. We acknowledge funding from Fundação de Amparo à Pesquisa de Estado de São Paulo, FAPESP, and Conselho Nacional de Desenvolvimento Cientitífico e Tecnológico, CNPq.

[1] J. Putschke for the STAR collaboration, Nucl.Phys. A783, 507 (2007), J. Phys. G34, S679 (2007).

[2] M. P. McCumber for the PHENIX Collaboration, J. of Phys. G35, 104081 (2008).

[3] M.J. Horner for the STAR Collaboration, J. of Phys. G34, S995 (2007).

[4] W.Lei for the PHOBOS collaboration, J. of Phys. G35, 104142 (2008); B. Alver et al (PHOBOS Collaboration) arXiv:0903.2811.

[5] A.Dumitru et al. Nucl. Phys. A810, 91 (2008).

[6] S.Gavin et al. Phys. Rev. C79, 051902 (2009).

[7] J.L. Nagle, Nucl. Phys. A830, 147C, (2009).

[8] M. Daugherity for the STAR Collaboration, J. of Phys. G35, 104090 (2008).

[9] B. Alver et al. (PHOBOS Collaboration), arXiv:0812.1172

[10] B.I.Abelev et al. (STAR collaboration) arXiv:0909.0191.

[11] S.A.Voloshin Phys.Lett.B632, 490 (2006).

[12] J. Takahashi, B.M. Tavares, W.L. Qian, R. Andrade, F. Grassi, Y. Hama, T. Kodama, and N. Xu, arXiv:0902.4870.

[13] H.J. Drescher, F.M. Liu, S. Ostapchenko, T. Pierog and K. Werner, Phys. Rev. C65, 054902 (2002).

[14] Y. Hama, T. Kodama and O. Socolowski Jr., Braz. J. Phys. 35, 24 (2005).

[15] R. Andrade, F. Grassi, Y. Hama, T. Kodama and O. Socolowski, Jr., Phys. Rev. Lett. 97, 202302 (2006).

[16] Y.Hama, R.P.G. Andrade, F. Grassi and W.-L.Qian, arXiv:0911.0811.

[17] A. Adare et al. (PHENIX Collaboration), Phys. Rev. C 78, 014901 (2008); Phys. Rev. C77 011901(R) (2008).

[18] A.Feng (for the STAR collaboration) J. Phys. G35 104082 (2008).

[19] B. I. Abelev (for the STAR Collaboration) J. Phys. G35 044010 (2008).

[20] S. Afanasiev et al. (PHENIX Collaboration), Phys. Rev. Lett. 101, 082301 (2008).

[21] J.R.Konzer (for the STAR Collaboration) arXiv:0907.4617.

[22] B. I. Abelev et al. (STAR Collaboration) Phys. Rev. Lett. 102052302 (2009). 